\title{
Aesthetic dental practice: between the medical practice and the socio-economic factors
}

\section{Opinion}

With the development of the medical practice the dental practice become mainly about two aspects. The first one is the one directly to its medical and clinical origins consisting of treating disease, delivering therapies ${ }^{1,2}$ and implementing preventive protocols. The second, aspects, is more recent and nor necessary clinical. It is rather about the appearance. Indeed, the number of clinics specialized in the dental aesthetic has considerably increased within the recent years.

However, the question of how it influence the quality of the clinical dental care since we can have the best doctors "migrating" from the clinical care to the dental aesthetic due to the attractive salaries and the better social status especially in the developing countries where the economic situation of many professionals even dentists is very limited which pushes many of them to search for solution to improve their situations and switch to the aesthetic dental practice. The policies of the insurance companies have obviously a strong influence on the financial aspects related to the imbalances between the aesthetic dental practice and the clinical one and aesthetic dental care is in the majority of cases available only to riche individuals.

The moral aspect and the importance of the appearance and the ethical aspects of medical intervention to modify it will not be discussed herein and is left to be debated within a multicultural platform under the diver's regulations of each country.

\section{Conclusion}

Aesthetic dental practice is a reality ${ }^{3,4}$ (although the situation is not similar in the developing and the developed countries) with which policy makers, health care professionals. Health insurance companies and all the related organisms should deal with in order to both keep the dental clinical as the priority in term of health policy and also make the aesthetic dental practice more accessible and why not as accessible as the clinical care.

\section{Acknowledgements}

Abdelaziz Ghanemi is a recipient of a 2013 CAS-TWAS President's Postgraduate Fellowship.

\author{
Volume 4 Issue 2 - 2016
}

\author{
Abdelaziz Ghanemi',2,3 \\ 'Key Laboratory of Animal Models and Human Disease \\ Mechanisms of the Chinese Academy of Sciences \& Yunnan \\ Province, Kunming Institute of Zoology, China \\ ${ }^{2}$ Kunming College of Life Science, China \\ ${ }^{3}$ University of Chinese Academy of Sciences, China
}

Correspondence: Abdelaziz Ghanemi, Key Laboratory of Animal Models and Human Disease Mechanisms of the Chinese Academy of Sciences \& Yunnan Province, Kunming Institute of Zoology, Chinese Academy of Sciences, China, Email ghanemiabdelaziz@hotmail.com

Received: January 28, 2016 | Published: February 04, 2016

\section{Conflict of interest}

The author declares no conflict of interest.

\section{References}

1. Ghanemi A. How important is pharmacognosy for doctors and dentists? Saudi Dent J. 2015;27(1):1-2.

2. Ghanemi A. For dentists and doctors: The neglected concepts about the factors influencing the effects of drugs. The Saudi Dent J. 2015;28(1):1-

3. Albaker AM. Aesthetic dental practices by dental and prosthodontic practitioners in Riyadh, Saudi Arabia. King Saud University Journal of Dental Sciences. 2012;3(2):77-83.

4. Sarkis E. Color change of some aesthetic dental materials: Effect of immersion solutions and finishing of their surfaces. Saudi Dent J. 2012;24(2):85-89. 